\title{
The Influence of Spatial Familiarity on Landmark Salience Sensibility Based on Eye Tracking
}

\author{
Litao Zhu ${ }^{\mathrm{a}, \mathrm{b}, \mathrm{c}}$, Milan Konečnýd ${ }^{\mathrm{d}}$, Jie Shen ${ }^{\mathrm{a}, \mathrm{b}, \mathrm{c}, *}$, Zdeněk Stachoňd $^{\mathrm{d}}$, Hana Švedovád \\ ${ }^{a}$ Key Laboratory of Virtual Geographic Environment (Nanjing Normal University), Ministry of Education, Nanjing 210023, China; \\ Litao.Zhuu@outlook.com(L.Z.), shenjie@njnu.edu.cn (J.S.) \\ ${ }^{b}$ Jiangsu Center for Collaborative Innovation in Geographical Information Resource Development and Application, Nanjing \\ 210023, China; \\ ${ }^{c}$ School of Geography Science, Nanjing Normal University, Nanjing 210023, China \\ ${ }^{d}$ Department of Geography, Faculty of Science, Masaryk University, Brno, Czech Republic; konecny@geogr.muni.cz (M.K.), \\ zstachon@geogr.muni.cz.(Z.S.), 451557@mail.muni.cz(H.Š.) \\ * Corresponding author
}

Keywords: Spatial Familiarity, Landmark, Landmark Salience Sensibility, Eye Tracking, Cognition

\begin{abstract}
:
Landmarks play an important role in navigation especially when people in the familiar and unfamiliar environment. These landmarks are usually used for expressing their spatial knowledge. This prior knowledge is related to the spatial familiarity that the spatial knowledge is acquired by individuals as a function of their experience in the environment (Gale et al., 1990). Individuals prefers to take into highly account their familiar route within the environment. Furthermore, the route descriptions include the differed types of landmarks in familiar/unfamiliar routes (Lovelace et al. 1999). The user's level of spatial familiarity for people is a key factor of how they navigate in the environment (Savage et al., 2012). In human navigation or wayfinding, the dependency on familiar landmarks is the highest priority (Golledge, 2003).

Landmarks are more distinguishably salient and prominent than the another spatial features (Sorrows and Hirtle, 1999). Therefore, the landmark salience refers to how easily this prominent spatial feature can be regarded as a landmark (Raubal and Winter, 2002). The landmark salience mainly divided into visual, structural (important location), and cognitive (semantic). Visual salience refers to the visual characteristics of spatial features such as color contrast with surroundings. Some visual characteristics include facade areas, shapes, colors and other properties. Semantic salience refers to the spatial knowledge related features such as a cultural importance of building. Structural salience refers to the spatial features play an important role in the structure of the spatial environment.
\end{abstract}

The level of spatial knowledge of the individual also an important factor in landmark selection (Nuhn and Timpf, 2018). Hamburger and Röser (2014) investigated landmark semantics and showed that famous buildings were better recognized than unfamiliar ones. Quesnot and Roche (2015) found that the familiar individuals prefer the semantic landmarks in environment while the unfamiliar individuals prefer the landmark with the prominent visual and structural. In contrast, people unfamiliar with an environment prefer landmarks with outstanding visual and structural characteristics.

The existing literature studies the method that landmark indicators to be considered are not comprehensive while can't reflect on the landmark characteristics. The reason is that the method of calculation and extraction for landmarks can't conform the individual preference needs of navigation. Compared with the traditional subjective evaluation method, the eye-tracking method provide objectivity and reliability. For example, a mobile eye tracking system was used by Kiefer et al. (2014) to investigate the process of self-localization. They discovered that test persons directed their attention longer on landmarks on the map and aligned them with objects in the surroundings during successful localization of their own position. Schwarzkopf et al. (2013) used fixed and mobile eye tracking devices to study that participates selected the navigation landmarks in the virtual environment and the real-world of the airport. In general, there are an only limited number of works related to the influence of spatial familiarity on landmark by using eye tracking method.

At present, many navigation systems are providing users with route directions under different travel modes. These navigation systems have the "distance-to-turn" method in route directions, however the landmark knowledge has not been conformed to user's habits and spatial cognitive in wayfinding. In order to solve these problems, this paper aims to study the characteristics of the user's spatial familiarity and landmark salience evaluation method in navigation. The following questions are addressed in this study: 1) What's the relationship between the spatial familiarity with the landmark selection in navigation for individuals? 2) What is the quantitative evaluation model to connect between the spatial familiarity with the landmark salience by using the eye-tracking data?

In our experiments, we will use eye tracking to explore the influence of spatial familiarity on landmark salience. The experimental path is part of the campus area of Nanjing Normal University. The subjects are divided into novice 
students and senior students from Nanjing Normal University as unfamiliar and familiar groups respectively. The procedures are divided into two parts, namely, the subjects should walk the experimental path within the fixation time and then they are asked to wear VR eye tracking module a Glass DK II special for HTC view (China; https://www.7invensun.com/) to do three types of tasks about landmark picture with the characteristics of visual, semantic and structural. With this research, we expect to establish a landmark salience evaluation method through eye movement data and analyse the landmark selection rules of groups with different spatial familiarity. It is helpful to design the pedestrian navigation system based on landmarks, improve the pertinence and reliability of navigation services and reduce the cognitive load for users.

\section{References}

Gale, N. , Golledge, R. G. , Pellegrino, J. W. , Doherty, S. (1990). The acquisition and integration of route knowledge in an unfamiliar neighborhood. Journal of Environmental Psychology, 10(1), 3-25.

Golledge, R. G. (2003). Human wayfinding and cognitive maps. In The Colonization of Unfamiliar Landscapes: The Archaeology of Adaptation (pp. 25-43). Routledge Taylor \& Francis Group.

Hamburger, K. , Röser, Florian. (2014). The role of landmark modality and familiarity in human wayfinding. Swiss Journal of Psychology, 73(4), 205-213.

Kiefer, P., Giannopoulos, I., Raubal, M. (2014). Where am I? Investigating map matching during self - localization with mobile eye tracking in an urban environment. Transactions in GIS, 18(5), 660-686.

Lovelace, K. L., Hegarty, M., Montello, D. R. (1999, August). Elements of good route directions in familiar and unfamiliar environments. In International conference on spatial information theory (pp. 65-82). Springer, Berlin, Heidelberg.

Nuhn E., Timpf S. (2018). Towards Personalized Landmarks. In: Fogliaroni P., Ballatore A., Clementini E. (eds) Proceedings of Workshops and Posters at the 13th International Conference on Spatial Information Theory (COSIT 2017). COSIT 2017. Lecture Notes in Geoinformation and Cartography. Springer, Cham

Quesnot T., Roche S. (2015). Quantifying the Significance of Semantic Landmarks in Familiar and Unfamiliar Environments. In: Fabrikant S., Raubal M., Bertolotto M., Davies C., Freundschuh S., Bell S. (eds) Spatial Information Theory. COSIT 2015. Lecture Notes in Computer Science, vol 9368. Springer, Cham

Raubal M., Winter S. (2002). Enriching Wayfinding Instructions with Local Landmarks. In: Egenhofer M.J., Mark D.M. (eds) Geographic Information Science. GIScience 2002. Lecture Notes in Computer Science, vol 2478. Springer, Berlin, Heidelberg

Savage, N. S., Chun, W., Chavez, N. E., Höllerer, T. (2013). Seems Familiar: An Algorithm For Inferring Spatial Familiarity Automatically. Computer Science Department, University of California, Santa Barbara, accessed Feb, 5.

Schwarzkopf, S., von Stülpnagel, R., Büchner, S. J., Konieczny, L., Kallert, G., Hölscher, C. (2013, September). What lab eye tracking tells us about wayfinding a comparison of stationary and mobile eye tracking in a large building scenario. In Eye Tracking for Spatial Research, Proceedings of the 1st International Workshop (in conjunction with COSIT 2013) (pp. 31-36).

Sorrows, M. E., Hirtle, S. C. (1999, August). The nature of landmarks for real and electronic spaces. In International Conference on Spatial Information Theory (pp. 37-50). Springer, Berlin, Heidelberg. 